\title{
Investigation on the Height Calculation of Water Flowing Fracture Zone during Coal Mining under Ordovician Limestone Nappe Aquifer
}

\author{
Chengcheng Chu \\ Department of Earth and Environment, Anhui University of Science and Technology, Huainan, Anhui 232001, China \\ Correspondence should be addressed to Chengcheng Chu; chu_cheng_cheng@126.com
}

Received 14 December 2021; Accepted 4 February 2022; Published 23 February 2022

Academic Editor: Yonghong Wang

Copyright (C) 2022 Chengcheng Chu. This is an open access article distributed under the Creative Commons Attribution License, which permits unrestricted use, distribution, and reproduction in any medium, provided the original work is properly cited.

\begin{abstract}
This paper focused on the height calculation of water flowing fracture zone during mining under the Ordovician limestone nappe aquifer. According to the critical conditions of crack arresting under the action of compression-shear stress, a formula for calculating the height of water flowing fracture zone during mining under the Ordovician limestone nappe aquifer was proposed, which takes tectonic stress, the influence of fault, the mechanical effect of the seepage field on the stress field, and the rock softening with water into consideration. The formula was applied to calculate the height of water flowing fracture zone above panel E3213 in the first eastern mining area of the Qianyingzi coalmine. The comparison between the theoretical calculation and the measured result shows that the formula proposed in this paper is closest to the measured value and further improves the calculation accuracy compared with the previous formulas. This study not only has important theoretical and practical significance for the safe mining of Qianyingzi coalmine, but also has important theoretical and practical significance for the coalmine facing the double threat of Ordovician limestone confined aquifer and reverse fault.
\end{abstract}

\section{Introduction}

The North China coalfield occupies a very important position in the distribution pattern of coal resources in China at the present stage, with abundant reserves. Especially under the action of thrust nappe structure in the southwest strong compression deformation area, the deep coal resources have great potential. However, due to thrust fault, the Ordovician limestone aquifer was pushed to the above coal roof in this area, which has characteristics of karst fissure development and good water abundance. In this case, the Ordovician limestone aquifer becomes a great threat to coal mining.

As we all know, coal seam mining will cause the movement and destruction of strata above the working face. When the mining depth reaches a certain depth $(100 \mathrm{~m})$, the movement of strata above the stope can be divided into caving zone, fault zone, and bending subsidence zone from bottom to top, as shown in Figure 1. The height of water flowing fracture zone refers to the sum of the caving zone and the fault zone, which is an important index to analyze the movement and deformation of overlying rock and predict roof water inrush.

Researchers have conducted many studies to predict the development height of water flowing fracture zone in stope, including theoretical calculation, numerical simulation, physical experiments, and other methods [1-22]. In the early 1980 s, on the basis of the measured data of coal mines in North China, Liu Tianquan academician put forward an empirical formula for the height of water flowing fracture zone. However, the formula has certain requirements for the depth and thickness of the coal seam mining, which is mainly applicable to the height calculation of the water flowing fracture zone of the thin coal seam with small mining depth (less than $300 \mathrm{~m}$ ). With the increasing depth of coal mining in China, the applicability of the formula has been greatly restricted. At present, the formula of water flowing fracture zone widely used by field technical staff of 


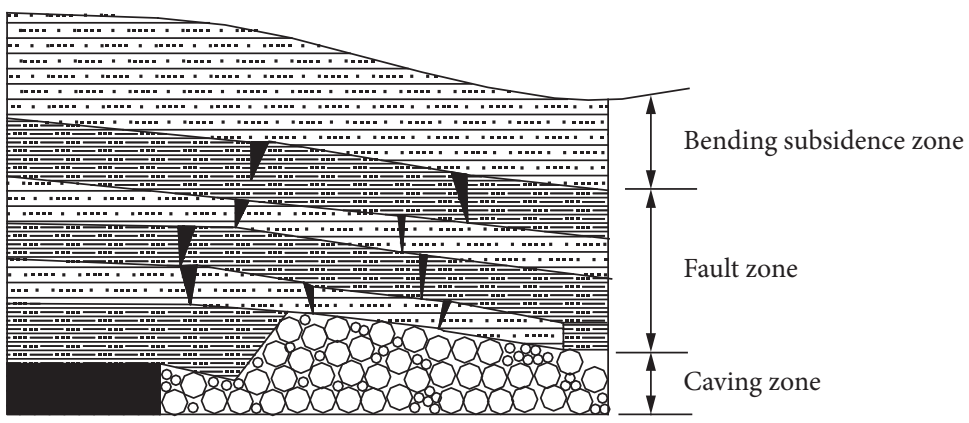

Figure 1: “Three-Zone” model of rock mass movement.

coal mines in China is mainly based on the experiential formula proposed in "Procedures for coal pillar reservation and coal mining of buildings, water bodies, railways and main roadways" [23]. As shown in Table 1, these formulas only consider the influence of coal seam dip angle, roof lithology of coal seam, and mining thickness of coal seam on the development height of water flowing fracture zone. Therefore, there is a certain gap between the calculated results and the actual height.

Due to the limitation of traditional empirical formulas, some scholars have studied the height calculation of water flowing fracture zone in recent years and put forward many new calculation methods. Based on the division theory of "four zones" of roof, Liu et al. [24] pointed out that the rock stratum above the separation zone is in an elastic bending state, and the height of the separation zone can be regarded as the maximum height of water flowing fracture zone. It is concluded that the expression of water flowing fracture zone height is

$$
h \approx z=\frac{1}{\eta}\left(1+C_{x} L_{0}-\frac{\tau}{\gamma H}\right),
$$

where $h$ is the height of the water flowing fracture zone; $z$ is the height of the separation zone; $C_{x}$ is the rock beam transfer coefficient; $L_{0}$ is the working face span; $\gamma$ is the weighted average weight of the roof rock stratum; $H$ is the buried depth of the unbroken rock stratum; $\tau$ is the maximum shear strength of the unbroken rock stratum; $\eta$ is the peak attenuation coefficient of the roof.

Equation (1) shows that the mining depth of coal seam and the working face span are the important factors controlling the development height of roof water flowing fracture zone.

Based on equation (1), Shi et al. [25] pointed out that, under the condition of large mining depth, $\tau / \gamma H$ tends to 0 , and $1+C_{X} L_{0} \approx C_{X} L_{0}$. Therefore, the calculation formula of the height of water flowing fracture zone can be expressed as

$$
h=\frac{C_{X} L_{0}}{\eta} \text {. }
$$

Equation (2) shows that the development height of water flowing fracture zone is proportional to the working face span under the condition of large mining depth.

In addition, considering the influence of seepage field, Shi et al. [25] further deduced the development height of water flowing fracture zone under the condition of large mining depth and the coupling of seepage field and stress field. The formula is as follows:

$$
h=\frac{k}{\eta k_{w} \sigma_{0}-p} L_{0}
$$

where $p$ is the water pressure of aquifer; $k_{w}$ is the softening coefficient of rock; $\sigma_{0}$ is the compressive strength of rock without water; $k$ is the proportion coefficient.

According to equation (3), the higher the water pressure in aquifer is, the higher the development height of the water flowing fracture zone will be. In addition, under the same geological conditions, the height of water flowing fracture zone under the coupling effect of seepage field and stress field is larger than that under the condition of only considering the stress field.

Based on the in-depth study of the influence law of key overburden layers on the development height of water flowing fracture zone, $\mathrm{Xu}$ et al. [26] proposed that only when the position of key layer is less than a certain critical height from the mining coal seam, the breaking fracture of key layer will develop into water flowing fracture, and the critical height of breaking fracture of key layer can be estimated by 7 to 10 times of mining thickness.

These research results have laid an important theoretical foundation for the research of this paper. However, there are few formulas for calculating the development height of water flowing fracture zone, which comprehensively consider the influence of geological structure characteristics and fluid-solid coupling. In this paper, according to critical conditions of crack arresting under the action of compression-shear stress, and from the perspective of the evolution of the crack propagation of the damaged rock mass, a formula for calculating the height of water flowing fracture zone with mining under a double threat from both the fault and roof confined water was proposed, which takes tectonic stress, the influence of fault, the mechanical effect of the seepage field on the stress field, and the rock softening with water into consideration.

\section{Critical Conditions of Crack Growth under the Action of Compression-Shear Stress}

Due to different stress conditions, cracks can be divided into three types: open crack (type I), slip crack (type II), and tear crack (type III), as shown in Figure 2. 
TABLE 1: Experiential computing formula of the height of water flowing fracture zone from the "Procedures for coal pillar reservation and coal mining of buildings, water bodies, railways and main roadways."

\begin{tabular}{|c|c|c|c|c|}
\hline Dip angle of coal seam & Roof lithology & Compressive strength (MPa) & Formula (1) & Formula (2) \\
\hline \multirow{4}{*}{$0 \sim 54^{\circ}$} & Hard & $40 \sim 80$ & $100 \sum M / 1.2 \sum M+2.0 \pm 8.9$ & $30 \sqrt{\sum M}+10$ \\
\hline & Medium hard & $20 \sim 40$ & $100 \sum M / 1.6 \sum M+3.6 \pm 5.6$ & $20 \sqrt{\sum M}+10$ \\
\hline & Soft & $10 \sim 20$ & $100 \sum M / 3.1 \sum M+5.0 \pm 4.0$ & $10 \sqrt{\sum M}+5$ \\
\hline & Ultrasoft & $<10$ & $100 \sum M / 5.0 \sum M+8.0 \pm 3.0$ & - \\
\hline
\end{tabular}

$\Sigma M$ refers to cumulative production thickness.

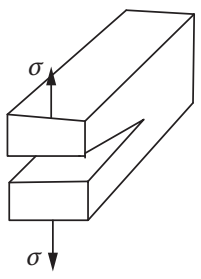

(a)

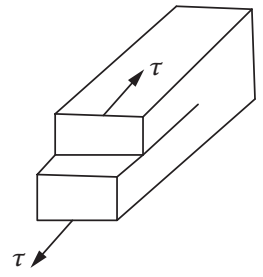

(b)

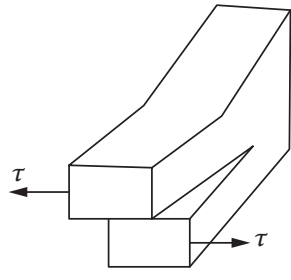

(c)

FiguRE 2: Three types of cracks. (a) Open crack; (b) slip crack; (c) tear crack.

According to the study of rock fracture mechanics, it is found that the geological rock mass is usually under compression, so the fracture is mostly compression-shear fracture [27]. A large number of experimental results and theoretical analysis show that the compressive shear crack begins to crack along the direction of maximum tensile stress, so the crack is propagated in type I $[28,29]$.

The theory of fracture mechanics puts forward the critical condition of crack instability propagation expressed by the stress intensity factor $\left(K_{I}\right)$ at the crack tip, also known as the $K$ criterion of brittle fracture. Stress intensity factor is a physical quantity that represents the strength of the stress field at the crack tip, which is determined by the load and the shape and size of the crack. The formula is as follows:

$$
K_{I}=K_{I C},
$$

where $K_{I}$ is the stress intensity factor at the crack tip; $K_{I C}$ is the plane strain fracture toughness of the material, which indicates the ability of the material to resist brittle fracture. It is a material constant and is generally measured by experiments.

As shown in Figure 3, there is a crack in the infinite plane (plane stress) under biaxial compression, with a length of $2 a$ and an azimuth of $\phi$. In the figure, $\sigma_{1}$ and $\sigma_{3}$ are maximum compressive stress and the minimum compressive stress, respectively. When the far-field stresses $\sigma_{1}$ and $\sigma_{3}$ increase to a certain value, the primary crack surface compresses and slides, and a branch crack formed at the tip. In order to simplify the calculation, the branch crack is idealized as a straight line with a length of $l$. When the primary crack surface slides, because the sliding displacement occurs only on the primary crack surface and the normal displacement is zero, only the driving force $\left(T_{s}\right)$ on the crack surface does work. For the branch crack, because it is only affected by the tensile force perpendicular to the crack propagation direction, the tangential displacement is zero and only the normal force $\left(T_{n}\right)$ does work.
If the primary crack and branch crack in Figure 3 are pulled straight to form a straight crack with a length of $2(a+l)$, the maximum sliding displacement on the primary crack surface $(\delta)$ can be regarded as the vector sum of the vertical displacement acting on the center of the straight crack $\left(\delta_{n}\right)$ and the shear displacement in the crack surface $\left(\delta_{s}\right)$. Their relationship can be expressed by the following formula:

$$
\delta^{2}=\delta_{n}^{2}+\delta_{s}^{2} .
$$

The type I stress intensity factors produced by $\delta_{n}$ at the center of the straight crack and $\delta_{s}$ at the tip of the branch crack are as follows:

$$
\begin{gathered}
K_{I}^{n}=\frac{B_{n} E_{0} \delta_{n}}{(l+a)^{1 / 2}}\left(B_{n}=0.4\right), \\
K_{I}^{s}=\frac{B_{s} E_{0} \delta_{s}}{(l+a)^{1 / 2}}\left(B_{s} \approx 1.0\right),
\end{gathered}
$$

where $K_{I}^{n}$ is the type I stress intensity factor produced by the vertical displacement acting on the center of the straight crack; $K_{I}^{s}$ is the type I stress intensity factor produced by the shear displacement in the crack surface; $E_{0}$ is the elastic modulus of rock mass.

According to the theory of fracture mechanics, the crack initiation is along the direction at an angle of $70.5^{\circ}$ with the primary crack. At this time, the stress intensity factor at the crack tip is $K_{I}^{s}$. However, when the crack propagation length is greater than $a$, the branch crack turns to the direction of the maximum principal stress, and the crack growth is dominated by the stress intensity factor $K_{I}^{n}$. This transformation can be multiplied by the factor $(1+L)^{1 / 2}(L=1 / a)$. This assumption has an impact on the results when $l$ is small and has little impact on the results when $l$ is large.

In conclusion, the total stress intensity factor at the crack tip can be expressed as 


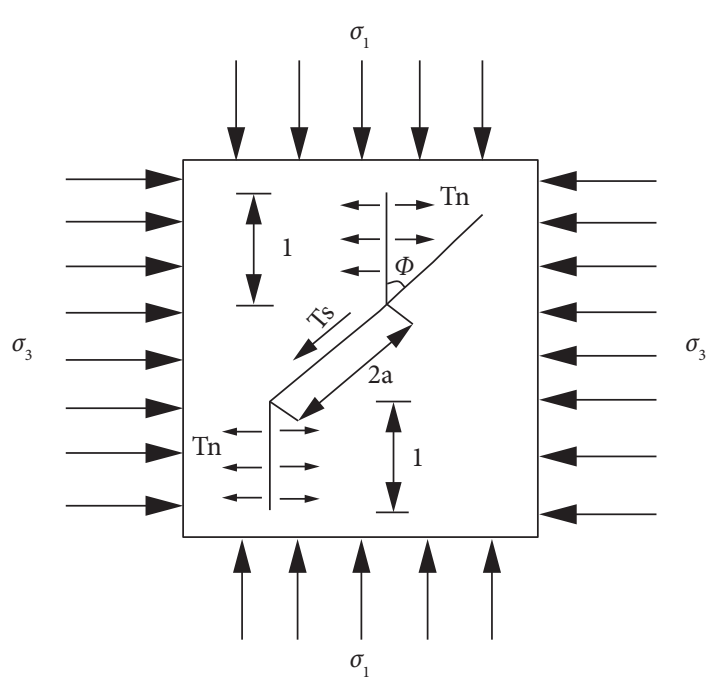

FIgURE 3: Branch crack propagation.

$$
\begin{aligned}
K_{I} & =K_{I}^{n}+K_{I}^{s}(1+L)^{1 / 2}, \\
& =\frac{\sqrt{\pi a}}{(1+L)^{3 / 2}}\left(\frac{2}{\sqrt{3}} T_{s}^{\text {(primary) }}+\frac{\sigma_{3} L}{B_{n}}\right)\left[B_{n} L+\frac{1}{(1+L)^{1 / 2}}\right],
\end{aligned}
$$

where $T_{s}^{\text {(primary) }}$ is the driving force on the primary crack surface.

According to the $K$ criterion of crack instability propagation in brittle materials, the critical condition for crack stop propagation under compressive shear stress is as follows:

$\frac{\sqrt{\pi a}}{(1+L)^{3 / 2}}\left(\frac{2}{\sqrt{3}} T_{s}^{(\text {primary })}+\frac{\sigma_{3} L}{B_{n}}\right)\left[B_{n} L+\frac{1}{(1+L)^{1 / 2}}\right]=K_{I C}$.

\section{The Formula for Calculating the Height of Water Flowing Fracture Zone under the Action of Stress Field}

Tectonic stress field refers to a local singular stress field produced by the interaction between a structure or a group of structures in the Earth and their forces. The direction of tectonic stress is horizontal or near horizontal, so it is also called horizontal stress field. The original rock stress in the tectonic stress field is concentrated and the horizontal stress increases. According to the measured results of global in situ stress, the horizontal stress in the tectonic development area is usually several times or even dozens of times the vertical stress [30, 31]. The tectonic stress field has obvious directionality and regionality.

Without considering the action of tectonic stress field, when the surface is nearly horizontal, the vertical stress $\left(\sigma_{V}\right)$ at any point in the rock mass is equal to the weight of the overburden.

$$
\sigma_{V}=\gamma H
$$

where $\gamma$ is the gravity of the rock, and $H$ is the buried depth.
The corresponding horizontal stress is

$$
\sigma_{h}=\lambda \gamma H=\frac{\mu}{1-\mu} \gamma H .
$$

where $\lambda$ is the static lateral pressure coefficient of rock; $\mu$ is Poisson's ratio of the rock.

For most hard rock masses, $\lambda=0.25 \sim 0.43$; that is, the horizontal stress is about $1 / 3 \sim 4 / 5$ of the vertical stress. However, the existence of faults will cause great differences between local stress field and regional stress field, and the lateral pressure coefficient of local stress field around reverse faults $\lambda>1$.

According to the distribution law of stope support pressure after coal seam mining [32], the maximum value of roof rock support pressure can be expressed as

$$
\sigma_{h}=K \gamma H
$$

where $K$ is the peak coefficient of bearing pressure of roof rock stratum; $H$ is the height of unbroken rock stratum; $\gamma$ is the weighted average gravity of roof rock stratum.

The peak coefficient of bearing pressure of roof rock stratum $(K)$ decreases linearly with the increase of roof normal height based on the coal seam roof interface $(Z)$, and its expression is

$$
K=K I-\eta Z,
$$

where $\eta$ is the peak attenuation coefficient of roof, which is mainly affected by mining thickness and roof lithology combination; $K I$ is the peak concentration factor of bearing pressure.

According to the practical mine pressure control theory [33], the relationship between the peak concentration factor of bearing pressure and the inclined length of working face is

$$
K^{\prime}=1+L_{0} C_{x}
$$

where $L_{0}$ is the inclined length of working face; $C_{x}$ is the transfer coefficient of rock beam.

By combining equations (11)-(13), the relationship between the maximum bearing pressure of roof rock and the height of water flowing fracture zone can be obtained. The expression is as follows:

$$
\sigma_{h}=\left(1+L_{0} C_{x}-\eta h\right) \gamma H,
$$

where $\sigma_{h}$ is the maximum bearing pressure of roof rock; $h$ is the height of water flowing fracture zone.

According to the fracture strength criterion of rock mass, when the peak stress of roof rock mass $\left(\sigma_{h}\right)$ is equal to the fracture strength of rock mass $\left(\left[\sigma_{1}\right]\right)$, the height of water diversion fracture zone reaches the maximum.

Then the expression of the maximum height of the development of the water flowing fracture zone in stope is

$$
h=\frac{1}{\eta}\left[1+L_{0} C_{x}-\frac{\left[\sigma_{1}\right]}{\gamma H}\right] \text {. }
$$

When the primary fracture or mining fracture in the roof rock is closed or filled, there is a reverse shear stress (friction resistance) between the two walls of the fracture. According 
to the Mohr Coulomb strength criterion, the reverse shear stress $\left(\tau_{0}\right)$ is

$$
\tau_{0}=f \sigma+C=\sigma_{1} \tan \phi_{0}+C_{0},
$$

where $\phi_{0}$ and $C_{0}$ are the original cohesion and friction angle of rock, respectively.

At this time, the driving force on the primary fracture surface is

$$
T_{s}^{(\text {Primary })}=\frac{\sigma_{1}-\sigma_{3}}{2} \sin 2 \phi-\sigma_{1} \tan \phi_{0}-C_{0},
$$

and substituting equation (17) into equation (8) we can obtain the following expression:

$\sigma_{1}=\frac{K_{I C} / \sqrt{\pi a} /(1+L)^{3 / 2}\left[B_{n} L+1 /(1+L)^{1 / 2}\right]+(2 / \sqrt{3}) C_{0}}{1-\lambda / \sqrt{3} \sin 2 \phi+L / B_{n} \lambda-2 / \sqrt{3} \tan \phi_{0}}$.

Substituting equation (18) into equation (15) we can obtain the maximum height of water flowing fracture zone under the action of stress field.

$$
h=\frac{1}{\eta}\left\{1+L_{0} C_{x}-\frac{K_{I C} / \sqrt{\pi a} /(1+L)^{3 / 2}\left[B_{n} L+1 /(1+L)^{1 / 2}\right]+2 / \sqrt{3} C_{0}}{\left[1-\lambda / \sqrt{3} \sin 2 \phi+L / B_{n} \lambda-2 / \sqrt{3} \tan \phi_{0}\right] \gamma H}\right\} .
$$

With the advancement of the working face to the fault direction, the fragmentation degree of the roof rock mass of the coal seam continues to increase and the compressive strength of the rock decreases. Because $K_{I C}=Y \sigma_{I C} \sqrt{\pi a}\left(\sigma_{I C}\right.$ is the critical compressive strength of rock fracture) for the infinite plane, $Y=1$, the fracture toughness of the rock also decreases. Considering the influence of fault on rock fracture toughness, equation (19) is modified as follows:

$$
h=\frac{1}{\eta}\left\{1+L_{0} C_{x}-\frac{t K_{I C} / \sqrt{\pi a} /(1+L)^{3 / 2}\left[B_{n} L+1 /(1+L)^{1 / 2}\right]+(2 / \sqrt{3}) C_{0}}{\left[1-\lambda / \sqrt{3} \sin 2 \phi+L / B_{n} \lambda-2 / \sqrt{3} \tan \phi_{0}\right] \gamma H}\right\} .
$$

$t$ represents the reduction coefficient of rock fracture toughness (rock strength) under the influence of fault, which can be obtained from the test data of borehole core.

\section{The Formula for Calculating the Height of Water Flowing Fracture Zone under the Action of Seepage and Stress Fields Coupling}

4.1. Coupling Mechanism of Seepage Field and Stress Field. The deformation and failure of overlying strata in stope are affected not only by mine pressure and mining effect, but also by seepage field.

The influence of stress field on seepage field is mainly because the stress field controls the pore change of porous medium or the geometric shape change of discontinuity in fractured medium, which affects the hydraulic characteristics of medium; the influence of seepage field on stress field includes mechanical action, physical action, and chemical action [24, 34]. Among them, the mechanical action of groundwater on rock mass includes hydrostatic pressure and hydrodynamic pressure. Hydrostatic pressure is the normal stress acting on the fracture structural plane, which belongs to surface force. The hydrostatic pressure per unit area is

$$
p_{w}=\gamma_{w} H_{1}
$$

where $\gamma_{w}$ is the gravity of water; $H_{1}$ is the head height.

Hydrodynamic pressure, also known as osmotic pressure, is a kind of volumetric force due to the hydraulic gradient of groundwater. Under the condition of homogeneous isotropy, the hydrodynamic pressure per unit volume is

$$
D=\gamma_{w} J
$$

where $J$ is the hydraulic gradient.

Literature [35] points out that the mechanical effect of seepage on the stress field of rock mass is that the seepage volume force is generated from the macropoint of view, and from the micropoint of view, the seepage pressure increases the stress intensity factor at the crack tip in rock mass, which has the effect of splitting. When the stress intensity factor at the crack tip reaches the critical value, the crack penetrates, propagates, and destroys, which reduces the stability of rock mass.

Physical action will have a great impact on the physical and mechanical properties of rock mass. This impact first depends on the mineral composition of rock and then depends on the composition, existing form, and content change of structural water in minerals. Chemical action includes chemical erosion, softening, and redox. When the rock mass contains soluble salt minerals, the seepage flow may dissolve and take them away, form cavities, or reduce the cohesion and shear strength in the rock mass; when the content of clay minerals in rock mass, such as shale, mudstone, and clayey siltstone, is high, the softening effect of fluid is more prominent, and the compressive strength will be reduced after the rock absorbs water.

The derivation of the calculation formula in this paper will focus on the mechanical action and the softening effect 
of water on rock when considering the influence of seepage field on stress field.

\subsection{Calculation Formula for the Height of Water Flowing} Fracture Zone. When there is seepage pressure $p$ in the fracture, assuming that the rock block does not conduct water, considering the mechanical effect of seepage field on rock mass and the softening effect on rock, the development height of roof water flowing fracture zone under fluid-solid coupling is analyzed.

The relationship between the effective normal stress of fracture surface $\sigma$ and $\sigma_{1}$ is

$$
\sigma=\sigma_{1}-p
$$

where $\sigma$ is the effective normal stress of fracture surface; $\sigma_{1}$ is maximum compressive stress; $p$ is the seepage pressure in the fracture.
According to the mine pressure control theory (Song 1998), the rock beam transfer coefficient is related to the strength of coal seam and the strength and movement of rock beam itself.

$$
C_{x}=\beta \frac{\sigma_{\text {coal }}}{\sigma_{\text {rock }}}
$$

where $\beta$ is the scale factor; $\sigma_{\text {caol }}$ is coal seam strength; $\sigma_{\text {rock }}$ is rock beam strength.

Considering the softening effect of water on rock, the compressive strength of rock under fluid-solid coupling is $\sigma_{\text {rock }}{ }^{\prime}=b \sigma_{\text {rock }}$, where $b$ is the softening coefficient of rock, which is an indicator of rock resistance to water softening. The smaller the softening coefficient of rock, the stronger the softening of rock.

The maximum height of water conducting fracture zone under the coupling action of seepage field and stress field is

$$
h=\frac{1}{\eta}\left\{1+L_{0} \beta \frac{\sigma_{\text {coal }}}{b \sigma_{\text {rock }}}-\frac{t b K_{I C} / \sqrt{\pi a} /(1+L)^{3 / 2}\left[B_{n} L+1 /(1+L)^{1 / 2}\right]+(2 / \sqrt{3}) C_{0}-p}{\left[1-\lambda / \sqrt{3} \sin 2 \phi+L / B_{n} \lambda-2 / \sqrt{3} \tan \phi_{0}\right] \gamma H}\right\}
$$

where $t$ is the reduction coefficient of rock fracture toughness (rock strength) under the influence of faults, which can be obtained from the test data of drilled cores; $\sigma_{\text {caol }}$ is coal seam strength; $\sigma_{\text {rock }}$ is rock beam strength.

It can be seen that, under the coupling action of seepage field and stress field, the greater the water pressure of aquifer, the greater the development height of water flowing fracture zone; the greater the softening coefficient of rock, the better the water resistance of rock, and the smaller the development height of water conducting fractured zone.

When the geological conditions and mining methods of the stope are certain, the development height of water flowing fracture zone is directly proportional to the mining depth of the coal seam and the inclined length of the working face. Therefore, reasonably determining the mining depth of coal seam and the inclined length of working face is an effective measure to control the development of water flowing fracture zone.

\section{Case Study}

E3213 working face is the trial mining working face in the first eastern mining area of Qianyingzi coalmine. Qianyingzi coalmine is located in the southwest of Suzhou City, Anhui Province, and it is a stratigraphic type of Huaibei coalfield, belonging to North China stratigraphic category. The mining depth of $3_{2}$ coal seam in the first eastern mining area of Qianyingzi coalmine is -280 to $-650 \mathrm{~m}$; the average thickness of $3_{2}$ coal seam is $3.3 \mathrm{~m}$. Drilling in mining area reveals that the strata from the bottom to the top are Ordovician, Carboniferous, Permian, Neogene, and Quaternary. As shown in Figure 4, DF200 fault pushes the Ordovician limestone to the top of $3_{2}$ coal seam directly, which is a reverse fault in research area. The dip angle of
DF200 fault is about $45^{\circ}$. The roof lithology of $3_{2}$ coal seam is interbed of soft and hard strata. The results of pumping experiments in the Ordovician limestone aquifer show that the aquifer has a hydraulic connection with the surrounding aquifer and is a medium water-rich aquifer. Therefore, $3_{2}$ coal seam mining is threatened by both DF200 fault and Ordovician limestone aquifer.

According to the drilling data, when E3213 working face is pushed $130 \mathrm{~m}$, the measured height of water flowing fracture zone is $72.05 \mathrm{~m}$. The mining depth of the coal seam in the working face is $420 \mathrm{~m}$, and the water pressure of the roof confined aquifer is $6.22 \mathrm{MPa}$. According to the main physical and mechanical parameters of each rock stratum in the mining area, the weighted average method is used to calculate that the average weight of the overlying rock stratum of the coal seam is $2.2 \times 10^{4} \mathrm{~N} / \mathrm{m}^{3}$, internal friction angle $\varphi_{0}=35^{\circ}$, cohesion $=5.95 \mathrm{MPa}$, and rock softening coefficient $b=0.9$. According to the test data of the compressive strength of the drill core, the ratio of the compressive strength of the rock in the fracture zone to the compressive strength of the complete core is $0.33 \sim 0.75$, that is, $t=0.33 \sim 0.75$, and the average value is 0.54 . According to the measured results of in situ stress in Qianyingzi coalmine, the lateral pressure coefficient of rock in the first eastern mining area is 1.65 , that is, $\lambda=1.65$. According to the analysis of the test data of drill core, the plane strain fracture toughness of roof rock $\left(K_{I C}\right)$ is $59.43 \mathrm{MPa} \mathrm{m}^{1 / 2}$. According to the data of the mining area, most of the fractures of the roof rock stratum are $0.2 \mathrm{~m}$, which refers to $a=2 \mathrm{~m}$ and $l=5 \mathrm{~m}$.

According to statistics and similar simulation experiments [36], when the mining height is $3 \sim 7 \mathrm{~m}$ and the working face span is $100 \sim 200 \mathrm{~m}$, the values of various coefficients between roof rock beams are $C_{x}=1 \sim 1.75$ and $\eta=2 \sim 3.5$. 


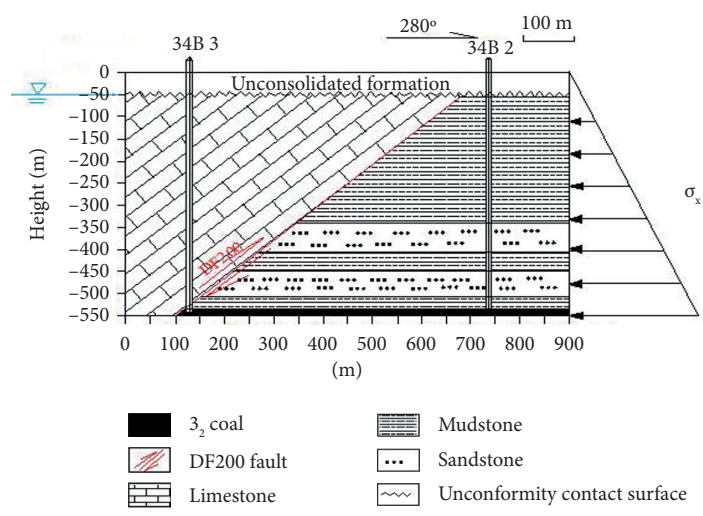

Figure 4: Mining hydrogeological and engineering geological model in the study area.

Based on this, this paper takes $C_{x}=1$ and $\eta=2$ to calculate the development height of water flowing fracture zone considering different influencing factors and the results are shown in Table 2 . The different conditions corresponding to No. 1-5 in the table are as follows: (1) only the influence of the self-weight stress field being considered; (2) considering the influence of confined aquifer; (3) considering the influence of tectonic stress field; (4) considering the influence of tectonic stress field and confined aquifer; (5) confined aquifer, tectonic stress field, and the influence of the fault on rock fracture toughness being considered.

As can be seen from Table 2:

(1) Compared with group 1, the theoretical values of the height of the water flowing fracture zone obtained by group 2, group 3, group 4, and group 5 increased by $15.2 \%, 18.9 \%, 31.6 \%$, and $32.6 \%$, respectively. Therefore, the water pressure of confined aquifer, fault, and tectonic stress promote the development of water conducting fracture zone.

(2) By comprehensively comparing the theoretical values obtained from the five groups of calculation schemes with the measured values of the height of the water diversion fracture zone in stope, the calculation result of scheme 5 is closest to the actual value. Therefore, the height of water flowing fracture zone of coal seam mining under Ordovician nappe aquifer should comprehensively consider the influence of confined aquifer water pressure, tectonic stress field, and fault.

In addition, using the empirical formula (1) and empirical formula (2) for calculating the height of water flowing fracture zone in medium hard roof slate given in Table 1, it can be obtained that the development height of water flowing fracture zone in overburden of E3213 working face is $30.1 \sim 41.3 \mathrm{~m}$ and $44.6 \mathrm{~m}$, respectively. Because the influencing factors considered in the traditional empirical formulas are relatively simple, the theoretical value of the development height of the water flowing fracture zone of the coal seam under the Ordovician limestone nappe aquifer calculated by these formulas is less than the actual value. If the risk assessment of water inrush from the stope roof is carried out based on these calculated values, the possible
TABlE 2: Statistics of the height of water flowing fracture zone.

\begin{tabular}{lcccc}
\hline Number & $\begin{array}{c}\text { Pressure of } \\
\text { confined } \\
\text { aquifer } p \\
(\mathrm{MPa})\end{array}$ & $\begin{array}{c}\text { Rock lateral } \\
\text { pressure } \\
\text { coefficient } \lambda\end{array}$ & $\begin{array}{c}\text { Influence } \\
\text { coefficient of } \\
\text { fault on rock } \\
\text { fracture } \\
\text { toughness } t\end{array}$ & $\begin{array}{c}\text { Height of } \\
\text { water } \\
\text { flowing } \\
\text { fracture } \\
\text { zone } h(\mathrm{~m})\end{array}$ \\
\hline 1 & 0 & 0.25 & 1 & 54.65 \\
2 & 6.22 & 0.25 & 1 & 62.96 \\
3 & 0 & 1.65 & 1 & 64.20 \\
4 & 6.22 & 1.65 & 1 & 71.94 \\
5 & 6.22 & 1.65 & 0.54 & 72.45 \\
\hline
\end{tabular}

risks will be ignored and the safety production of the coal mine will be affected.

\section{Conclusions}

A formula for calculating the height of water flowing fracture zone during mining under the Ordovician limestone nappe aquifer was proposed. The formula was on the basis of critical conditions of crack arresting under the action of compression-shear stress and from the perspective of the evolution of the crack propagation of the damaged rock mass, taking tectonic stress, the influence of fault, the mechanical effect of the seepage field on the stress field, and the rock softening with water into consideration. The derived formula for calculating the height of water flowing fracture zone during mining under the Ordovician limestone nappe aquifer was used to calculate the height of water conducting fractured zone of panel E3213 under different conditions. Under the gravity stress field, the calculated value was $54.65 \mathrm{~m}$. Taking the confined aquifer into consideration, the calculated value was $62.96 \mathrm{~m}$ and taking tectonic stress into consideration, the calculated value was $64.20 \mathrm{~m}$. Considering influences of both tectonic stress and confined aquifer, the calculated value was $71.94 \mathrm{~m}$. Taking tectonic stress, confined aquifer, and the influence of fault on rock fracture toughness into comprehensive consideration, the calculated value was $72.45 \mathrm{~m}$. The results show that these three factors could promote the growth of water conducting fractured zone. The comparison between the theoretical calculation and the measured result shows that the formula proposed in this paper is closest to the measured value and further 
improves the calculation accuracy compared with the previous formulas. Therefore, the height of water flowing fracture zone of coal seam mining under Ordovician nappe aquifer should comprehensively consider the influence of confined aquifer water pressure, tectonic stress field, and fault.

\section{Data Availability}

The data used to support the findings of this study are available from the corresponding author upon request.

\section{Conflicts of Interest}

The authors declare no conflicts of interest.

\section{Acknowledgments}

This work was supported by the Natural Science Foundation of Anhui Science and Technology Department of China under Grant no. 1908085QE248 and the Natural Science Foundation of Anhui Education Department of China under Grant no. KJ2018A0100. Many faculty members at the School of Resources and Geosciences, China University of Mining and Technology, reviewed the manuscript and provided helpful comments. Their support is gratefully acknowledged.

\section{References}

[1] W. H. Sui, "Safety geology and geological education for mining engineers," in Proceedings of the International Symposium and 9th Asian Regional Conference of the International Association for Engineering Geology and the Environment, Beijing, China, September 2013.

[2] C. He, W. Lu, W. Zha, and F. Wang, "A geomechanical method for predicting the height of a water-flowing fractured zone in a layered overburden of longwall coal mining," International Journal of Rock Mechanics and Mining Sciences, vol. 143, Article ID 104798, 2021.

[3] M. Alber, R. Fritschen, M. Bischoff, and T. Meier, "Rock mechanical investigations of seismic events in a deep longwall coal mine," International Journal of Rock Mechanics and Mining Sciences, vol. 46, no. 2, pp. 408-420, 2009.

[4] G. F. Hofmann and L. J. Scheepers, "Simulating fault slip areas of mining included seismic tremors using static boundary element numerical modeling," Mining Technology, vol. 120, no. 1, pp. 53-64, 2011.

[5] J. G. Ni, J. Wang, Y. L. Tan, and Q. Xu, "Mechanical mechanism of overlying strata breaking and development of fractured zone during close-distance coal seam group mining," International Journal of Mining Science and Technology, vol. 30, pp. 207-215, 2020.

[6] W. P. Huang, Y. F. Gao, B. Wang, and J. R. Liu, "Evolution rule and development height of permeable fractured zone under combined-strata structure," Journal of Mining and Safety Engineering, no. 2, pp. 330-335, 2017.

[7] C. C. Chu, Investigation on the Responses of Overburden Stress and Water Pressure to Mining under the Ordovician Nappe, China University of Mining and Technology, Xuzhou, China, 2016.
[8] B. B. Yang, J. Liu, X. Zhao, and S. Zheng, "Evaporation and cracked soda soil improved by fly ash from recycled materials," Land Degradation \& Development, vol. 32, no. 9, pp. 2823-2832, 2021.

[9] B. B. Yang, D. Li, S. Yuan, and L. Jin, "Role of biochar from corn straw in influencing crack propagation and evaporation in sodic soils," Catena, vol. 204, Article ID 105457, 2021.

[10] B. B. Yang, S. Yuan, Y. Liang, and J. Liu, "Investigation of overburden failure characteristics due to combined mining: case study, Henan Province, China," Environmental Earth Sciences, vol. 80, p. 143, 2021.

[11] B. B. Yang, S. T. Du, X. M. Zhao, D. Tang, and C. Yang, "Decision making of curriculum attainment degree for engineering geology based on fuzzy set theory," Advances in Civil Engineering, vol. 2021, Article ID 1743778, 6 pages, 2021.

[12] B. B. Yang, Z. P. Zhang, W. L. Ma, M. Hu, and Y. Zhang, "Effect of tea waste on cracking of foundation soil," Advances in Materials Science and Engineering, vol. 2021, Article ID 7525811, 2021.

[13] B. B. Yang, K. Xu, and Z. Zhang, "Mitigating evaporation and desiccation cracks in soil with the sustainable material biochar," Soil Science Society of America Journal, vol. 84, no. 2, pp. 461-471, 2020.

[14] B. X. Yuan, Z. H. Li, Z. Q. Zhao, and H. Ni, "Experimental study of displacement field of layered soils surrounding laterally loaded pile based on transparent soil," Journal of Soils and Sediments, vol. 21, no. 9, pp. 3072-3083, 2021.

[15] B. X. Yuan, Z. H. Li, Z. L. Su, Q. Luo, M. Chen, and Z. Zhano, "Sensitivity of multistage fill slope based on finite element model," Advances in Civil Engineering, vol. 2021, Article ID 6622936, 13 pages, 2021.

[16] B. X. Yuan, Z. H. Li, Y. Chen et al., "Mechanical and microstructural properties of recycling granite residual soil reinforced with glass fiber and liquid-modified polyvinyl alcohol polymer," Chemosphere, vol. 286, Article ID 131652, 2021.

[17] B. X. Yuan, M. Sun, Y. X. Wang, and L. Zhai, "Full 3D displacement measuring system for 3D displacement field of soil around a laterally loaded pile in transparent soil," International Journal of Geomechanics, vol. 19, no. 5, Article ID 04019028, 2019.

[18] B. X. Yuan, M. Sun, L. Xiong, Q. Luo, S. P. Pradhan, and H. Li, "Investigation of $3 \mathrm{~d}$ deformation of transparent soil around a laterally loaded pile based on a hydraulic gradient model test," Journal of Building Engineering, vol. 28, no. 6, Article ID 101024, 2019.

[19] B. Bai, R. Zhou, G. Q. Cai, W. Hu, and G. Yang, "Coupled thermo-hydro-mechanical mechanism in view of the soil particle rearrangement of granular thermodynamics," Computers and Geotechnics, vol. 137, no. 8, Article ID 104272, 2021.

[20] B. Bai, Q. K. Nie, Y. K. Zhang, X. Wang, and W. Hu, "Cotransport of heavy metals and $\mathrm{SiO}_{2}$ particles at different temperatures by seepage," Journal of Hydrology, vol. 597, Article ID 125771, 2021.

[21] B. Bai, G. C. Yang, T. Li, and Y. Gao-Sheng, "A thermodynamic constitutive model with temperature effect based on particle rearrangement for geomaterials," Mechanics of Materials, vol. 139, Article ID 103180, 2019.

[22] Y. Wu, J. Cui, J. Huang, and W. Zhang, "Correlation of critical state strength properties with particle shape and surface fractal dimension of clinker ash," International Journal of Geomechanics, vol. 21, no. 6, Article ID 04021071, 2021. 
[23] State Coal Industry Administration, Procedures for Coal Pillar Reservation and Coal Mining of Buildings, Water Bodies, Railways and Main Roadways, China Coal Industry Press, Beijing, China, 2017.

[24] X. L. Liu, Study on the Theory and Application of Water Rock Coupling Process and its Multi-Scale Behavior, Tsinghua University, Beijing, China, 2008.

[25] L. Q. Shi, H. Q. Xin, P. H. Zhai et al., "Calculating the height of water flowing fracture zone in deep mining," Journal of China University of Mining and Technology, vol. 41, no. 1, pp. 37-41, 2012.

[26] J. L. Xu, W. B. Zhu, and X. Z. Wang, "New method to predict the height of fractured water-conducting zone by location of key strata," Journal of China Coal Society, vol. 37, no. 5, pp. 762-769, 2012.

[27] S. M. Yi and D. Z. Zhu, Introduction to Damage Mechanics of Fractured Rock Mass, Science Press, Beijing, China, 2005.

[28] M. F. Ashby and S. D. Hallam, "The failure of brittle solids containing small cracks under compressive stress states," Acta Metallurgica, vol. 34, no. 3, pp. 497-510, 1986.

[29] H. Horii and S. Nemat-Nasser, "Compression-induced microcrack growth in brittle solids: axial splitting and shear failure," Journal of Geopgysical Research, vol. 90, no. B4, pp. 3105-3125, 1985.

[30] S. R. Su, Influence of Fault Structure on In-Situ Stress Field and its Engineering Significance, Chengdu Institute of Technology, Chengdu, China, 2001.

[31] Q. D. Xie, Characteristics of Tectonic Stress Field in Fault Area and its Influence on Tunnel Surrounding Rock Stability, Central South University, Changsha, China, 2010.

[32] M. G. Qian, W. P. Shi, and J. L. Xu, Mining Pressure and Strata Control, China University of mining and Technology Press, Xuzhou, China, 2010.

[33] Z. Q. Song, Practical Mine Pressure Control Theory, China University of mining and Technology Press, Xuzhou, China, 1998.

[34] L. Q. Shi and J. Han, Mechanism and Prediction of Floor Water Inrush, China University of mining and Technology Press, Xuzhou, China, 2004.

[35] D. Z. Zhu and H. X. Guo, Hydraulic Foundation of Fractured Rock Mass, Science Press, Beijing, China, 2007.

[36] M. D. G. Salamon, "Behaveior and design of coal pillars," Australia Coal Journal, vol. 32, no. 32, pp. 11-22, 1991. 